\title{
Facilitating remote access to specialist medical expertise through the scaled up adoption of a smartphone application: A South African case
}

\author{
N Blanckenberg, ${ }^{1}$ MMed (Fam Med), MBA HCL; T Motala, ${ }^{2}$ BSc Eng (Chem), MBA \\ ${ }^{1}$ Cape Winelands District, Western Cape Government Health, South Africa \\ ${ }^{2}$ University of Stellenbosch Business School, Cape Town, South Africa
}

Corresponding author: N Blanckenberg (natasha.blanckenberg@westerncape.gov.za)

\begin{abstract}
Background. In the context of a shortage of medical specialists, a medical referral app, designed for use on smartphones, was launched in 2014 for use by doctors in the public health service in South Africa.

Objectives. As this is a novel intervention, with potential to have an impact on the use of scarce resources, and because not much was known about the use of the app, a descriptive study was undertaken to assess its adoption in Western Cape Government Health (WCGH) facilities. Methods. Usage data of the app in WCGH facilities, in terms of referral and user numbers, were obtained from the date of its introduction in 2014. In addition, all the referrals to WCGH facilities for July 2019, stripped of any identifying data of patients or doctors, were analysed for origin, destination, outcome and response times. Descriptive statistics were used to analyse the data.

Results. Use of the app grew rapidly from 40 referrals per quarter to 16437 per quarter after 5 years in use, with a cumulative total of 95381 referrals. In July 2019, active users of the system included 913 sending doctors and 298 receiving doctors, representing 20 medical specialties. The senders and receivers were representative of every level in the healthcare system, from clinic to tertiary hospital. In July 2019, a total of 5941 referrals were sent by means of the app to public facilities in Western Cape Province. Of the referrals, $80 \%$ were classified as acute and $20 \%$ as non-urgent. The referral outcomes included $51 \%$ accepted for transfer, $19 \%$ accepted for a specialist appointment, and $13 \%$ concluded with advice alone without the need for a specialist appointment or patient transfer - this category accounted for $28 \%$ of non-urgent referrals and $9 \%$ of acute referrals. In $50 \%$ of referrals, advice was given to the referring doctor, either as an additional or the only outcome. The median response times were 9 minutes for acute referrals and 19 minutes for non-urgent referrals.

Conclusions. This study documents the scale-up of a mobile phone consultation and referral app from pilot phase to significant growth in use across a resource-constrained healthcare system. In a large proportion of cases, advice was given to the referring doctor by means of the app, frequently obviating the need for a specialist appointment or patient transfer. This finding demonstrates that a mobile app has the potential to reduce the need for face-to-face specialist visits, thereby improving the use of scarce medical resources.
\end{abstract}

S Afr Med J 2022;112(2):108-112. https://doi.org/10.7196/SAMJ.2022.v112i2.15799

The public health service in South Africa (SA), serving $85 \%$ of the population, is based on a primary healthcare (PHC) model, ${ }^{[1]}$ which is in accordance with international best practice. ${ }^{[2]}$ Primary care providers (PCPs) are supported by teams of specialists at referral hospitals. However, the public health sector in SA is under-resourced and beset by organisational inefficiencies. ${ }^{[3]}$ Access to specialist care is increasingly difficult owing to the inadequate number of specialists to serve the needs of the population. ${ }^{[4,5]}$

Referral systems have a significant impact on healthcare systems and the use of resources, and have therefore been extensively studied. ${ }^{[6]}$ Extant studies have focused mainly on demand management interventions to decrease the numbers of referrals, to prevent unnecessary referrals of problems that could have been resolved at primary care level, and to improve the quality or preparation of the referrals received ${ }^{[6-9]}$ There has recently been an increase in the use of secure web-based electronic referral systems in an effort to facilitate and improve referrals. ${ }^{[10]}$ Many studies have shown a reduction in avoidable, inappropriate and incomplete referrals, and high levels of acceptability to those sending and receiving referrals by means of electronic referral systems. ${ }^{[11-13]}$ These studies were conducted in well-resourced settings such as the USA, Canada, the UK and Europe. Studies of electronic referral systems in low-resource settings have largely been confined to pilot projects. ${ }^{[14]}$ However, with the emergence of mobile technologies, there have been studies describing projects piloting the use of mobile phone technology to facilitate specialist referrals in low-resource settings ${ }^{[15,16]}$ Doctors in resourceconstrained settings, often without easy access to computers at their work stations, widely use instant messaging applications (primarily WhatsApp) as an informal technological aid to the primary-specialist care interface. ${ }^{[15]} \mathrm{A}$ secure mobile referral application, Vula, has been available to doctors working in public healthcare in SA since 2014, starting with one specialty and growing gradually in scope and in uptake. ${ }^{[17]}$

In late 2018, Western Cape Government Health (WCGH), a government body that provides healthcare for 6.5 million people, awarded a tender for an electronic service to facilitate referrals to medical specialists throughout the provincial health service (WCGH, circular H13 of 2019, communication: Vula Mobile e-referral app). WCGH provides healthcare through a network of 268 PHC facilities (including clinics and community health centres), 34 district hospitals, and 5 regional, 3 psychiatric and 3 tertiary referral hospitals. ${ }^{[18]}$ Referrals to specialist departments have historically been arranged primarily by means of telephone calls. Other referral channels in use include email, web portals and the unofficial use of direct messaging 
applications, such as WhatsApp (WCGH, circular H13 of 2019).

There is a lack of research on the use of information and communication technology solutions for referrals on a large scale in resource-constrained settings, as few projects make it out of the pilot phase. ${ }^{[19]}$ In addition, there is a lack of research on the use of mobile devices to facilitate referrals on a large scale in any context. These knowledge gaps, combined with the potential of a smartphonebased referral application to positively impact on the use of scarce healthcare resources, provided good reasons to study the use of a novel mobile referral app in the context of the primary-specialist care network of a large public health service. As not much was known about the use of the app, a descriptive study was required before impact studies could be undertaken.

\section{Methods}

Ethics approval was granted by the Research Ethics Committee: Humanities of Stellenbosch University (ref. no. 10958). Institutional approval to use the referral data was obtained from WCGH and from Vula Mobile.

A descriptive quantitative study was undertaken. Data were extracted from the Vula Mobile database (a PostgreSQL relational database) with scripts written specifically for these queries and exported as Excel spreadsheets. Longitudinal usage data of the app in Western Cape Province, in particular referral and user numbers, were obtained from the introduction of the app in 2014 until July 2019. In addition, all the Vula referrals to WCGH facilities for July 2019, stripped of any identifying data of patients or doctors, were obtained. Referrals to WCGH facilities were included regardless of whether they originated in a public or private healthcare facility. Referrals were analysed by a family physician for origin, destination and response times. In addition, each referral was categorised according to its level of acuity. A referral was categorised as acute if the patient's condition was imminently life threatening or disabling, if the patient was considered to be a danger to himself or others, or if the referring doctor requested transfer of the patient to the referring hospital. The case was judged to be non-urgent if the referring doctor requested an outpatient appointment or requested advice for a condition that was not imminently life threatening or disabling. When the acuity of the case was not obvious from reading the referral, it was classified as indeterminate.
The outcome of each interaction was determined and categorised, and these categories were then grouped into broader categories. The transfer category consisted of patients accepted for immediate transfer, internal referrals or down-referrals (patients accepted to a lower level of care), and patients placed on a waiting list for a bed. The appointment category included cases where the receiving doctor advised the referring doctor to arrange an outpatient appointment for a patient or a date was provided for an outpatient appointment or procedure. The advice only category included cases where the receiving doctor provided advice on the diagnosis or management of the patient, but did not arrange to see the patient in person. The rejected/withdrawn category included cases where the patient required a specialist visit, but the doctor who received the referral did not agree to see the patient. This category also included cases where the referring doctor withdrew the referral because the patient refused referral, absconded or died. The no response category included the messages where no response could be detected. However, it did not include internal referrals (within the same building) with no recorded response, where it could reasonably be expected that a response was made in person, so these cases were categorised with the internal referrals in the accepted category. The incomplete category was created to mitigate the potential categorising error for referrals where there was a response, but the interaction between doctors did not appear to reach a conclusion.
This may have been because the interaction resumed outside of the app, e.g. by means of a telephone call or instant messaging conversation.

Descriptive statistics were used to analyse the data.

\section{Results}

The Vula Mobile electronic consultation and referral (eCR) application originated in the Western Cape and commenced operations in April 2014, initially receiving referrals to one specialty, ophthalmology. Fig. 1 demonstrates the number of referrals sent per quarter to WCGH facilities. Referrals increased from 40 in the first quarter to 16437 in the last quarter under review, after 5 years of operation. The cumulative total of referrals sent by means of Vula to WCGH during this period was 95381 .

Out of a total of 5941 referrals sent to WCGH facilities in July 2019, the facility of origin of $8.9 \%$ of referrals $(n=526)$ could not be determined with certainty because of doctors who had moved between facilities and had not updated their Vula profiles. Of the remaining 5415 referrals of certain origin, $71.8 \%(n=3890)$ were sent from non-specialist facilities, including private practices, clinics, community health centres (CHCs) and smaller district hospitals. Of these referrals sent from nonspecialist facilities, 198 were sent from private sector non-specialist facilities to public facilities.

The $28.2 \%$ of referrals ( $n=1525$ ) that were sent from specialist units represented

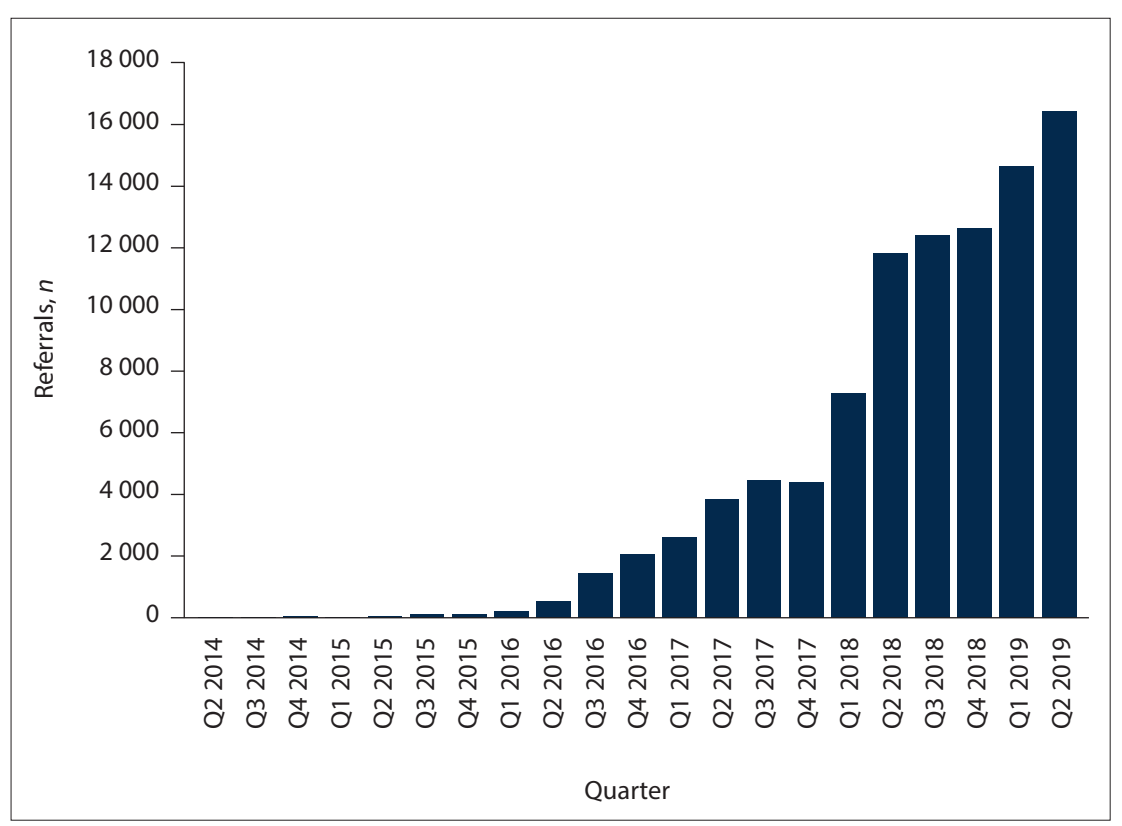

Fig. 1. Number of referrals sent by means of Vula Mobile to facilities operated by Western Cape Government Health, per quarter, 2014 - 2019. (Q1, Q2, Q3, Q4 = quarters 1, 2, 3 and 4.) 
18 sending specialties, with $63.8 \%$ of these referrals originating from the specialty of emergency medicine.

In July 2019, referrals were made by means of Vula by 913 different users, of whom 289 made only one referral each. The busiest referrer, registered at a clinic in a deprived urban area, made 63 referrals. The median (interquartile range (IQR)) number of referrals made per user was $3(1-8)$.

Referrals were received by 75 different units, representing 20 medical specialties in 17 facilities.

Most referrals (94.8\%) were received at specialist hospitals of all levels: tertiary, regional, and large district hospitals with specialist units, as indicated in Table 1 . The $5.2 \%$ of referrals that were received at non-specialist facilities were either at specialist services hosted in non-specialist facilities or at a district hospital emergency centre that was not led by an emergency medicine specialist (and therefore not classified as a specialist unit).

As shown in Table 2, referrals were received by 20 different specialties, with emergency medicine leading by a large margin. Orthopaedic surgery received the second highest number of referrals, well ahead of other specialties.

Of the 74 specialist units that received referrals, 11 received only one. The busiest unit, an emergency medicine unit covering both a large district hospital and a CHC, received 724 referrals. The median (IQR) number of referrals received per unit was 10 (3 - 93).

Referrals were received by 298 doctors. Forty-eight of the doctors who received referrals received only one for the month, while the busiest doctor, working in orthopaedic surgery in a large urban district hospital, received 181 referrals by means of Vula in one month. The median (IQR) number of referrals received per doctor was 11 (3 - 27).

\begin{tabular}{ll}
$\begin{array}{l}\text { Table } \mathbf{1} \text { Referrals received according to the level of the } \\
\text { receiving facility }\end{array}$ \\
\hline Level of facility receiving referrals & $\begin{array}{l}\text { Referrals } \\
\text { received, } \boldsymbol{n}(\%)\end{array}$ \\
\hline Large district hospital with specialist units & $2994(50.4)$ \\
Tertiary hospital & $1922(32.4)$ \\
Regional hospital & $573(9.6)$ \\
District hospital & $298(5.0)$ \\
Psychiatric hospital & $121(2.0)$ \\
Tuberculosis hospital & $22(0.4)$ \\
Community health centre & $11(0.2)$ \\
Total & 5941
\end{tabular}

Table 2. Percentage of the total number of referrals received in July 2019 , per specialty

\begin{tabular}{ll}
\hline Specialty receiving referrals & Referrals received, $\boldsymbol{n}$ (\%) \\
\hline Emergency medicine & $2298(38.7)$ \\
Orthopaedic surgery & $1272(21.4)$ \\
General surgery & $662(11.1)$ \\
Ophthalmology & $500(8.4)$ \\
Dermatology & $310(5.2)$ \\
Internal medicine & $233(3.9)$ \\
Psychiatry & $166(2.8)$ \\
ENT & $105(1.8)$ \\
Other & $395(6.7)$ \\
Total & 5941 \\
ENT = ear, nose and throat. &
\end{tabular}

A summary of referral outcomes according to case acuity is presented in Table 3. The category designated as transfer could be subdivided into patients who were accepted for immediate transfer to a referral unit $(80.0 \%)$, those referred internally within a facility (14.9\%), patients put on a waiting list for a bed (4.9\%), and down-referrals $(0.3 \%)$ - those referred from a higher to a lower level of care.

Of patients who were advised of the need for an appointment, $73.6 \%$ were given the appointment date by means of the app, and the rest were advised the further step of contacting the unit to arrange an appointment.

For $12.8 \%$ of referrals, the referring doctor received advice regarding the diagnosis and/or the management of the patient without arrangements being made for the patient to attend the specialist unit as either an in- or outpatient. This outcome of advice only was more common for non-urgent referrals than acute referrals. Advice only was a frequent outcome for dermatology ( $44.4 \%$ of total dermatology referrals), internal medicine (24.5\%), ophthalmology $(23.4 \%)$ and orthopaedic $(21.5 \%)$ referrals.

The reasons for referral rejection included recommendations to refer to a different specialty (31.0\%), a different level of care (26.2\%) or a hospital in a different area (7.6\%). Other reasons included a lack of inpatient capacity (8.8\%), outpatient clinics being fully booked for the year $(12.1 \%)$, the rejection of Vula as a channel for that type of referral (5.5\%), and confusion about which doctor was on call (4.8\%).

As well as the primary outcomes of referrals as described above, doctors frequently gave advice to referring doctors in addition to accepting them for transfer or giving an outpatient appointment. Of the 5506 referrals that received a response, 50.4\% included advice from the receiving doctor as the only or as an additional outcome. This advice related to the diagnosis and management of patients and navigation of the health system. For patients accepted for transfer, advice was given on the initial treatment required before transfer. In orthopaedic surgery referrals, this commonly included a review of the X-rays, advice on manipulation and immobilisation of the fracture, and a review of the post-reduction X-rays sent from the treating doctor to the doctor in the orthopaedic unit. For patients accepted for an appointment, advice was commonly given about the investigations to be done before the appointment date. Health systems advice was commonly given in the form of a review of the legal documentation required for involuntarily admitted psychiatric patients. The referring doctor was required to photograph and send completed documents to the psychiatrist for review, frequently resulting in corrections.

The time taken from when the referral was made to the first response from the receiving doctor ranged from less than a minute to 28 days, with a median (IQR) response time of 11 minutes overall (3 - 40). For acute referrals the range was from less than a minute to 18 days, with a median (IQR) response time of 9 (3 - 35) minutes. For non-urgent referrals, the response time ranged from less than a minute to 28 days, with a median (IQR) response time of $19(6-72)$ minutes.

\section{Discussion}

This study describes the use of a versatile mobile health (m-health) innovation in the patient referral process of a large public healthcare service. What makes Vula unique is that it is designed for use on smartphones, has been developed in a middle-income country, ${ }^{[11]}$ and has successfully grown out of the pilot phase, where many technological innovations in healthcare get stuck. ${ }^{[14,20]}$ Vula is, to the knowledge of the researchers, the only mobile eCR in largescale use at present in any context. Its use has been found to be 
Table 3. Referral outcomes according to case acuity

\begin{tabular}{lllll}
\hline Outcome & Acute, $\boldsymbol{n}(\%)$ & Non-urgent, $\boldsymbol{n}(\%)$ & Indeterminate, $\boldsymbol{n}(\%)$ & Total, $\boldsymbol{n}(\%)$ \\
\hline Transfer & $2997(63.0)$ & $31(2.7)$ & $1(5.9)$ & $3029(51.0)$ \\
Appointment & $582(12.2)$ & $563(48.3)$ & $1(5.9)$ & $1146(19.3)$ \\
Advice only & $437(9.2)$ & $321(27.6)$ & 0 & $758(12.8)$ \\
Rejected or withdrawn & $325(6.8)$ & $84(7.2)$ & $2(11.8)$ & $411(6.9)$ \\
No response & $299(6.3)$ & $128(11.0)$ & $8(47.1)$ & $435(7.3)$ \\
Incomplete & $119(2.5)$ & $38(3.3)$ & $5(29.4)$ & $162(2.7)$ \\
Total & $4759(80.1)$ & $1165(19.6)$ & $17(0.3)$ & 5941
\end{tabular}

widespread in terms of range of specialties, facilities and levels of care, and number of users. The app's versatility has been demonstrated by its role in facilitating access to care for both acute and non-urgent cases, unlike most eCRs that are used exclusively for non-urgent cases $^{[11]}$ and a few that are used exclusively for acute cases. ${ }^{[21,22]}$ Even though it is asynchronous in nature, Vula's suitability for acute referrals is demonstrated both by the good response times achieved by the receiving doctors and the dominance of emergency medicine as both a receiving and referring specialty.

Analysis of referrals has shown that Vula enables a number of interventions, the likes of which have previously been demonstrated to be associated with improved referral processes. It facilitates direct communication between the referring doctor and the relevant specialist, ${ }^{[23]}$ using an interactive medium that can share images and is compliant with privacy legislation. ${ }^{[24]}$ It provides case-based ongoing medical education and enables the screening of referrals for completeness, appropriateness and urgency. ${ }^{[9]}$ The extensive use of Vula's interactive consultation functionality, as documented in the present study, has allowed patients to be managed by PCPs, at the facilities where they presented for care, aided by input from specialists delivered by means of the app. The asynchronous nature of the medium allows receiving doctors to respond to requests timeously, but without the interruptions of telephone calls demanding immediate attention. In a large number of cases, consultation by means of the app removed the need for a face-to-face visit to a specialist unit, particularly for cases of a less urgent nature. The screening of referrals performed by means of Vula confirms its use as a demand management tool in the patient-orientated supply network. ${ }^{[7]}$ Its role in the screening of referrals provides an opportunity for the prevention of inappropriate, avoidable or inadequately prepared visits to specialist services, which has the potential to improve the use of scarce and costly specialist resources. ${ }^{[10]}$ This potential to reduce the need for in-person specialist consultations is especially valuable in the context of social distancing and restricted travel brought on by the COVID-19 pandemic.

Beyond its now documented roles in the provision of clinical care and demand management, Vula has another role, as demonstrated by this study. It provides a single database for the recording and analysis of referrals, referral patterns and the remote consultation workload throughout the service in which it operates. These data have until now not been available in healthcare systems, especially in those that do not use electronic medical records.

\section{Study limitations}

Owing to time and capacity constraints, this study was confined to referrals sent to WCGH, although Vula is used in other provinces, in the private sector and in Namibia. For the same reasons, the referral context outside of the use of the app, such as the number of specialist departments and users not using Vula for referrals, and the number of referrals made in total within the healthcare system, could not be included in the study.

The study, while describing the use of the app, does not address the impact of the app on service utilisation, waiting times and patient outcomes. It does not compare the app with other referral systems and tools. It also does not address cost-effectiveness, user experience or patient satisfaction, and does not cover the impact that the app has had on the professional relationships between the different levels of healthcare providers. The aspects not included in this study should be considered important areas of research for further study.

\section{Conclusions}

This study documents the scale-up of a locally developed smartphone referral and consultation application from pilot phase to use at a commercial scale in a resource-constrained healthcare system. This app enables direct communication between referring doctors and the doctors on call for particular specialist units for that day. It provides a secure and legally compliant platform for transferring detailed patient information and images, both enabling and archiving discussions between the referring and receiving doctors, without the need for extensive information technology infrastructure. These findings demonstrate significant ongoing growth in uptake by both referrers and specialist teams for both acute and non-urgent cases. In a large proportion of cases, advice was given to the referring doctor by means of the app, frequently obviating the need for a specialist visit. This study demonstrates that a smartphone app can be used as a referral, consultation and demand management tool. It can facilitate access to specialist input while reducing the need for face-to-face specialist appointments or patient transfers. Remote specialist input through use of a smartphone app has the potential to optimise the use of scarce specialist resources while improving patient care at the point of entry into the healthcare system.

Declaration. The research for this study was done in partial fulfilment of the requirements for NB's MBA HCL degree at Stellenbosch University. Acknowledgements. NB would like to thank the Discovery Foundation for the Rural Fellowship Award. The authors express their appreciation to Vula Mobile for granting access to their data.

Author contributions. NB and TM conceptualised the research topic and study design. NB took the lead in collecting data and writing the manuscript. TM supervised the study and provided critical feedback to shape the final manuscript.

Funding. NB received funding in the form of a Rural Fellowship Award from the Discovery Foundation. Discovery Foundation and the authors have no affiliation with Vula Mobile.

Conflicts of interest. None. 
1. Van Rensburg HC. Health and Health Care in South Africa. 2nd ed. Pretoria: Van Schaik Publishers, 2012.

2. Declaration of Astana. Global Conference on Primary Health Care, Astana, Kazakhstan, 25 - 26 October 2018. World Health Organization and UNICEF, 2018. https://www.who.int/docs/default-source/primaryhealth/declaration/gcphc-declaration.pdf (accessed 23 October 2018).

3. Begg K, Mamdoo P, Dudley L, Andrews G, Englebrecht J, Lebese L. Development of a national strategic ramework for a high-quality health system in South Africa. In: Rispel LC, Padarath A, eds. South African Health Review 2018. Durban: Health Systems Trust, 2018:77-85.

4. Econex. Identifying the determinants of and solutions to the shortage of doctors in South Africa: Is there a role for private medical education? August 2015. https://econex.co.za/publication/research-report-1/ (accessed 16 February 2019).

5. Wishnia J, Strugnell D, Smith A, Ranchod S. The Supply of and Need for Medical Specialists in South Africa. Cape Town: Percept, 2019.

6. Akbari A, Al-Alawi M, Grimshaw J, et al. Interventions to improve outpatient referrals from primary care to secondary care. Cochrane Database Syst Rev 2008, Issue 4. Art. No.: CD005471. https://doi. org/10.1002/14651858.CD005471.pub2

7. Blank L, Baxter S, Buckley Woods H, et al. Referral interventions from primary to specialist care: Review of international evidence. Br J Gen Pract 2014;64(629):e765-e774. https://doi.org/10.3399/bjgp14X682837

8. Senitan M, Alhaiti AH, Lenon GB. Factors contributing to effective referral systems for patients with non-communicable disease: Evidence-based practice. Int J Diabetes Dev Ctries 2018;38(March):115-123. https://doi.org/10.1007/s13410-017-0554-5

9. Greenwood-Lee J, Jewett L, Woodhouse L, Marshall D. A categorisation of problems and solutions to improve patient referrals from primary to specialty care. BMC Health Serv Res 2018;18(986):1-16. https:// improve patient referrals from prima
doi.org/10.1186/s12913-018-3745-y

10. Vimalanda V, Gupte G, Seraj S, et al. Electronic consultations (e-consults) to improve access to speciality . Vimalanda V, Gupte G, Seraj S, et al. Electronic consultations (e-consults) to improve access to speciality
care: A systematic review and narrative synthesis. J Telemed Telecare 2015;21(6):323-330. https://doi. care: A systematic review and

11. Liddy C, Drosinis P, Keely E. Electronic consultation systems: Worldwide prevalence and their impact on patient care - a systematic review. Fam Pract 2016;33(3):274-285. https://doi.org/10.1093/fampra/cmw024 2. Chen A, Murphy E, Yee H. eReferral - a new model for integrated care. N Engl J Med 2013;368(26):24502453. https://doi.org/10.1056/NEJMp1215594

13. Barnett M, Yee H, Mehrotra A, Giboney P. Los Angeles safety-net program eConsult program was rapidly adopted and decreased wait times to see specialists. Health Aff 2017;36(3):483-499. https://doi. org/10.1377/hlthaff.2016.1283
14. Delaigue S, Bonnardot L, Steichen O, et al. Seven years of telemedicine in Médecins Sans Frontières demonstrate that offering direct specialist expertise in the frontline brings clinical and educational value. J Glob Health 2018;8(2):1-9. https://doi.org/10.7189/jogh.08.020414

15. Den Hollander D, Mars M. Smart phones make smart referrals: The use of mobile phone technology Den Hollander D, Mars M. Smart phones make smart referrals: The use of mobile phone technology
in burn care - a retrospective case series. Burns 2017;43:190-194. https://doi.org/10.1016/j. in burn care -

16. Azfar RS, Lee RA, Castelosoccio L, et al. Reliability and validity of mobile teledermatology in human immunodeficiency virus-positive patients in Botswana: A pilot study. JAMA Dermato 2014;150(6):601-607. https://doi.org/10.1001/jamadermatol.2013.7321

7. Springhall T. Dr William Mapham: From the heart of rural health to the minds of the DoH. eHealt News, 2017. http://ehealthnews.co.za/dr-william-mapham/ (accessed 16 February 2019).

18. Western Cape Government Health. Annual Report 2017 - 2018. Cape Town: Western Cape Government, 2018. https://www.westerncape.gov.za/sites/www.westerncape.gov.za/files/wcgh_annual report 2018.pdf (accessed 1 October 2019).

19. Fanta G, Pretorius L. A conceptual framework for sustainable ehealth implementation in resourceconstrained settings. S Afr J Ind Eng 2018;29(3):132-147. https://doi.org/10.7166/29-3-2055

20. Agarwal S, Perry H, Long L, Labrique A. Evidence on feasibility and effective use of mHealth strategies by frontline health workers in developing countries: Systematic review. Trop Med Int Health 2015;20(8):1003-1014. https://doi.org/10.1111/tmi.12525

21. Mehet I, Sanchez Franco LC, Gascon Conde I, et al. The NORSe: Changing the way we communicate. Ann R Coll Surg 2018;100(3):161-164. https://doi.org/10.1308/rcsann.2018.0020

22. Jayashree M, Khandelwal N, Sharma M, Angrish S, Patil M. Feasibility of an e-referral system fo . Jayashree M, Khandelwal N, Sharma M, Angrish S, Patil M. Feasibility of an e-referral system for
streamlining referrals to pediatric emergency room of a tertiary care teaching hospital in North India: A preliminary report. J Pediatr Crit Care 2018;5(5):99-102. https://doi.org/10.21304/2018.0505.0043

3. De Vries J, Huijsman R. Supply chain management in health services: An overview. Supply Chai Manage 2011;16(3):159-165. https://doi.org/doi.org/10.1108/13598541111127146

24. South African Government. Protection of Personal Information Act 4 of 2014. https://www.gov.za/ documents/protection-personal-information-act (accessed 9 May 2019).

Accepted 12 October 2021. 